\section{Dust clouds and frictional generation of glow discharges on Mars}

A REMARKABLE characteristic of those samples of the martian soil which have so far been analysed is the absence of carbonaceous matter down to the parts per billion $\left(10^{\circ}\right)$ level $^{1}$. As well as a lack of endogenous organic material there is no sign of the component expected from infalling carbonaceous meteorites. The inorganic particles constituting the fine martian soil seem to be extremely "clean'-far cleaner than terrestrial desert or Antarctic analogues. I suggest here that glow discharges generated by friction within dust clouds might explain this apparent absence of carbonaceous matter. In addition glow discharges might account for some reactions noted in the Viking biological experiments.

Fine siliceous material is frequently raised by Martian winds to produce dust clouds, sometimes in enormous quantities sufficient to shroud the entire planet ${ }^{2}$. In the present cold, dry conditions ${ }^{3}$ it seems inescapable that frictional electrostatic charging will occur. On Earth we see natural examples of this triboelectric phenomenon in sandstorms and dusty volcanic eruptions ${ }^{3}$. Laboratory studies have confirmed that movement and dispersion of many types of finely-divided material result in the separation of charge and the generation of considerable electrostatic potentials ${ }^{4}$. It is not essential that the system be heterogeneous: homogeneous particles of different sizes can take up opposite polarities. The magnet experiments on the Viking landers have demonstrated a certain heterogeneity in the martian soil ${ }^{1}$, however. Separation of the particulate components of a charged cloud can produce oppositely charged regions within it. At the pressures existing in the lower terrestrial atmosphere the potentials may be equilibrated by a spark discharge - the most dramatic example being volcanic lightning ${ }^{5}$. At reduced pressures, however, charge equalisation is facilitated by glow discharge ${ }^{6}$. Experiments ${ }^{7}$ show this mechanism to be most effective in the pressure range $0.1-10$ torr: at the surface of Mars there is a dry $95 \%$ carbon dioxide atmosphere at a pressure of 5-6 torr (7-8 mbar; ref. 1). Studies of the glow discharge resulting from the generation of frictional electricity at low pressures were begun in the eighteenth century ${ }^{8}$, but frictional devices were soon supplanted by more reliable and controllable sources of high potential for the investigation of electrical discharges in gases. I therefore decided to check experimentally on the effect of agitating dry powders at reduced pressure.

A 5-1 Pyrex flask was rotated manually about a hollow stub axle, two O-rings providing a vacuum seal. The speed of rotation controlled the magnitude and form of agitation of the dust from gentle sliding to a continuous cascade, an oscillatory motion proving especially effective. Evacuation was accomplished through the hollow axle by a conventional vacuum system incorporating gauges, a gas bleed, and a two-stage rotary pump. With air as the gaseous medium and fine sand (previously washed and oven dried) as the particulate material it was found that discharges visible in a darkened room were readily excited over a wide range of reduced pressures. At 0.1 torr a faint greenish-white glow extended for more than $5 \mathrm{~cm}$ above the agitated sand, being somewhat more intense adjacent to the rising wall of the rotating vessel. The system was then isolated from the pump and air allowed to enter as required through a needle valve. In the pressure range of particular interest (4-6 torr) the continuous glow contracted and brightened, and was accompanied by long, forked, ribbon discharges about $1 \mathrm{~cm}$ wide darting intermittently through the cascade of dispersed grains. Occasional intense spots of light on the surface of the moving sand gave a 'twinkling' appearance. At 10 torr ribbon discharges became narrower and brighter, but less frequent. This progression continued until at 50 torr only bright twinkling discharges remained.

It is recognised that the glow discharge is a very effective scavenger of even the most tenacious traces of organic matter from surfaces (particularly silicates) because cleaning with such a discharge is the conventional final stage in preparing an as- tronomical mirror for aluminising 9 . The mechanism is primarily that of ion bombardment, but reactive intermediates produced in the gas phase may also be important ${ }^{10}$. It is therefore suggested that the fine, near-surface martian soil will have been subjected to repeated dissemination by seasonal winds and, while within these clouds, the separated particles exposed many times to the cleaning action of glow discharges. It will be noted that this process could operate discontinuously throughout an extended period of time right up to the present. It also differs from irradiation by solar ultraviolet in that the latter could not penetrate beyond the surface of a cloud or the undisturbed soil: the two mechanisms may be complementary.

In addition to the cleaning action, it is relevant to consider the accumulated chemical effects which may be produced by glow discharges ${ }^{7}$. It has been shown that they promote dissociation of molecular gases such as carbon dioxide, and can act as effective generators of ultraviolet light, ions, free radicals, atomic oxygen and ozone ${ }^{11,12}$. The last two in particular could persist for varying lengths of time after the discharge, and diffuse to subsurface locations in the grains to exert powerful oxidising effects. Potential products include peroxide bonds, percarbonates (which evolve oxygen and carbon dioxide on gentle warming, and oxygen on addition of liquid water) and hydrogen peroxide addition compounds ${ }^{1}$. It is therefore suggested that the widespread reddish coloration of the martian surface ${ }^{1}$, as well as the unexpected reactions observed in the Viking biology experiments ${ }^{13}$, could in part be due either directly or indirectly to glow discharges. Living microorganisms (including spore-formers) do not long survive exposure to glow discharges and their products ${ }^{12}$, so on this basis the fine, red, surficial martian dust would seem unfavourable for life-detection experiments. A better sampling scheme might be coring in the postulated permafrost terrain ${ }^{2}$. It is perhaps ironic that in different conditions (notably in the presence of water) glow discharges can promote the abiogenic synthesis of organic molecules?.

Finally, although it is not expected that glow discharges on Mars would be detectable from orbit (much less from Earth), it would seem worthwhile to attempt radiofrequency and electrostatic field measurements on future landings.

\section{A. A. Mills}

Departments of Geology and Astronomy, University of Leicester, University Road, Leicester, UK

Received 19 April; accepted 30 June 1977

Soffen, G. A. Science 194, $1274(1976)$

Mutch, T. A., Arvidson, R. E., Jones, K. L., Head, J. W., III \& Saunders, R. S. The Geology of Mars (Princeton University Press, Princeton, 1976).

Latham. J. Q. J/ R. Met. Soc. 90, 91 (1964).

Harper. W. R. Consact and Frictional Electrification (Oxford University Press, Oxford, 1967) Anderson, R. et al. Science 148, 1179 (1965).

Llewellyn Jones, F. The Glow Discharge (Methuen, London, 1966)

Blaustein. B. D. (ed.) (hemical Reactions in Electricat Discharges / Adr. in Chem. Series No. 80), American Chemical Soc., 1969).

Harvey, E. N. A History of Luminescence (American Philosophical Society, 1957).

Strong, J. Modern Physicat Lahoratory Practice (Prentice-Hall, Englewood Cliffs, 1938).

Holland. L. Vacum Deposition of Thin Films (Chapman and Hall, London, 1956).

meleus, H. J. \& Anderson, J. S. Modern Aspects of Inorganic Chemistry (Routledge and Kegan

1959).

13 Oyama, V. I., Berdahl, B. J. \& Carle, G. C. Nature 265, 110 (1977).

\section{Daytime ionosphere scintillation associated with geomagnetic storms}

EQUATORIAL spread F, although a night-time phenomena, has been reported on a few occasions during the dawn period'. This is thought to be due to the remains of irregularities produced during the night. Earlier results have also shown that ionospheric scintillations and spread $F$ have 